\title{
La motivación en el área de Expresión Plástica
}

\section{Motivation in the area of Plastic Expression}

\author{
INÉs LÓPEZ-MANRIQUE \\ Universidad de Oviedo. Asturias. España. \\ Departamento de Ciencias de la Educación. Área de Didáctica de la Expresión Plástica. \\ lopezines@uniovi.es \\ Juan Carlos San Pedro-Veledo \\ Universidad de Oviedo. Asturias. España. \\ Departamento de Ciencias de la Educación. Área de Didáctica de la Expresión Plástica \\ juancar@uniovi.es \\ Carmen GonzÁlez-GonzÁlez de Mesa \\ Universidad de Oviedo. Asturias. España. \\ Departamento de Ciencias de la Educación. Área de Didáctica de la Expresión Corporal. \\ gmcarmen@uniovi.es
}

Recibido: 23 de enero de 2013

Aceptado: 18 de noviembre de 2013

\section{Resumen}

El presente artículo desarrolla la cuestión de la motivación en el área de Expresión Plástica. El texto se divide en tres partes. En la primera de ellas nos centramos en explicar el mecanismo de la motivación y su presencia en el ámbito educativo. En la segunda abordamos la cuestión de la motivación en Educación Artística aportando los datos de estudios experimentales y las opiniones de expertos del área. Por último incluimos una síntesis de las estrategias y recursos más frecuentes para potenciar la motivación en Educación Artística, distinguiendo entre estrategias para el alumnado y el profesorado.

Palabras clave: Estrategias de Motivación; Educación Artística; Sistema Límbico; Motivación intrínseca; Motivación extrínseca.

López-Manrique, I. San Pedro-Veledo, J.C. González-González de Mesa, C. (2014): La motivación en el área de Expresión Plástica. Arte,Individuo y Sociedad, 26 (2) 199-213.

\footnotetext{
Abstract

The present paper develops the subject of motivation in the area of Artistic Expression Area. The text is divided into three parts.In the first one we explain the motivation mechanism and its presence in the field of education. In the second one we deal with the matter of motivation in Artistic Education providing information of experimental studies data and the opinions of experts on the area. Finally we include a summary of strategies and more frequent resources to promote motivation in Artistic Education, distinguishing between strategies for students and for teachers.

Keywords: Strategies of Motivation; Artistic Education; Límbic System; Intrinsic Motivation; Extrinsic Motivation 
López-Manrique, I. San Pedro-Veledo, J.C. González-González de Mesa, C. (2014): Motivation in the area of Plastic Expression. Arte,Individuo y Sociedad, 26 (2) 199-213.

Sumario: 1.Introducción, 2.La motivación, 2.1. La motivación en el estudiante, 2.2.Cerebro y motivación; 3.Motivación y Educación Artística, 3.1. Las motivaciones en el aula de Educación Artística frente a las motivaciones de la práctica artística externa al marco educativo, 3.2. Estudios cuantitativos y cualitativos sobre motivación y Educación Artística, 3.2.1. Investigaciones sobre la motivación en Educación Artística en relación con otras variables internas, 3.2.2. Algunas estrategias motivacionales habituales en el ámbito de la Educación Artística.4. Conclusiones. Referencias.

\section{Introducción}

Nos interesa particularmente abordar la cuestión de la motivación en el contexto de la Educación Artística porque en el ámbito educativo se considera la motivación como uno de los elementos fundamentales para que el alumno realice actividades y tareas educativas y sea constante para adquirir los conocimientos y destrezas que se precisen en las distintas disciplinas (Alonso, 1992). Pero nos encontramos que en el área de Expresión Plástica éste es un tema menos desarrollado que en otras áreas próximas como la Expresión Musical y la Expresión Corporal.

\section{La motivación}

Según González y Tourón (1992), la motivación está mediada entre el autoconcepto del individuo y la tarea a la que se tiene que enfrentar. Y la conducta humana está guiada por la representación de metas, a su vez estas metas son demostrar competencia y proteger o mejorar la autoestima.

La motivación del alumno surge al ponerse en contacto con las personas y todo tipo de material educativo que le acompaña en el centro de enseñanza, por tanto la motivación le origina un mejor aprendizaje y adquisición de conocimientos (Garrido, 1986).

Es importante para la motivación en educación la denominada Perspectiva Temporal Futura denominada P. T. F. (Nuttin, 1985). La cual va de la mano del logro y de la conducta dirigida a meta, incluye un aspecto cognitivo que es lo que se va a recibir a largo plazo por el trabajo realizado y para recorrer ese futuro precisa una motivación. Se ha comprobado que cuando el estudiante tiene un alto deseo de metas futuras el trabajo habitual es mayor (De Volder y col., 1982).

\subsection{La motivación en el estudiante}

Revisaremos los tipos de motivación en los estudiantes: la motivación intrínseca, la motivación extrínseca y la motivación cognitivo social.

La motivación intrínseca es el estado subjetivo que mueve la realización de una actividad sin ninguna recompensa externa. En el ámbito de la educación la curiosidad y el interés son los estímulos intrínsecos fundamentales. 
La curiosidad en el alumno (Berlyne, 1960), originará emociones positivas para así avanzar en el trabajo que realice; se encuentra unido a la creatividad, el estudiante en el tema que tenga que realizar preguntará, estudiará e investigará sobre él.

El interés (Super, 1964), puede ser medible por la cantidad de tiempo que una persona dedica a una actividad sin tener ninguna obligación y sin recibir nada a cambio por el trabajo. En el interés hay atención y gusto por el tema.

En el segundo tipo de motivación, la motivación extrínseca el individuo realiza un trabajo esperando una recompensa. Las conductas realizadas tienen como fin obtener algo atractivo a cambio como puede ser un premio, existiendo también conductas para alejarse de algo repulsivo, que sería un castigo (Reeve, 1994).

En el ámbito educativo la motivación extrínseca viene dada por premios y alabanzas.

Por último nos encontramos con la motivación cognitivo social, que es aquella en la cual el alumno en el medio educativo adquiere motivaciones como el logro y las metas de logro. La primera de ellas, motivación de logro se comprende como el interés para realizar un trabajo de forma excelente y estar destacado con respecto a otras personas.

En el estudiante se forma de la unión entre los cambios que va teniendo el alumno y los que tienen lugar en el medio educativo (Garrido, 1990). El niño ya se da cuenta de que el éxito o el fracaso pueden depender de él y reacciona con alegría o con vergüenza. En la motivación de logro escolar intervienen: la familia, los compañeros y los profesores.

Atkinson (1957), propone la teoría de la motivación de logro que conlleva la tendencia al éxito y la tendencia a evitar el fracaso. Esta teoría se fue modificando por otros autores y la persona además de pensar en el presente, debe de pensar en un futuro deseable (Reeve, 1994), que le lleve a una meta futura a largo plazo. La motivación de logro sería una actividad orientada hacia una meta denominada meta de logro. Según Nicholls (1984), en la motivación escolar se cree que el autoconcepto de la persona determina las metas que puede alcanzar en su motivación de logro. Parece claro que un autoconcepto positivo es importante para la persona y para la sociedad.

El segundo tipo de motivación cognitivo social es la meta de logro, Locke (1969), presenta su teoría del papel motivador de las metas específicas en el comportamiento del individuo en el mundo laboral, de tal forma que un trabajador que tenga las metas claras, en igualdad de condiciones con otro que no las tenga así de claras, siempre hará mejor el trabajo el que tiene las metas claras, como ya hemos citado anteriormente.

Las metas para lograr motivación según Locke, han de tener cierto grado de dificultad y cuando la meta supone un reto, dentro de unos límites razonables, las personas aportan mayor esfuerzo y atención; lo mismo ocurre en el ámbito educativo.

Las personas para alcanzar la meta de logro dirigimos nuestro comportamiento de una forma u otra, así una persona conoce su poder para realizar una tarea, lo demuestra y además lo compara con los demás (Nicholls, 1984, 1989).

Para elegir las metas de logro además de un componente personal como es el autoconcepto, interviene aquello que la situación requiera en un determinado momento para así considerar las metas más importantes (Ames y Archer, 1988). También afecta 
el tipo de meta que elige la persona: el profesor, algún compañero (los más significativos), el tipo de tareas y sobre todo aquellas que le permiten mejorar sus habilidades o adquirir otras nuevas (Ames, 1992).

\subsection{Cerebro y motivación}

La motivación al tener la propiedad de movernos y mantenernos para realizar una actividad, precisa energía para realizarla. Esta disposición para la acción, produce en el individuo un estado de vigilia, de estar alerta, se trata de un estado corporal con manifestaciones físicas que implican al sistema nervioso central, al sistema nervioso periférico y a los órganos por ellos inervados.

La motivación está relacionada con la afectividad y está íntimamente ligada a las emociones; si algo nos motiva está acompañado de emociones y las emociones también pueden motivarnos (Damasio, 1999). Las emociones son más específicas y están más influidas por una situación cercana reflejando la apreciación inmediata de nuestra relación con el medio ambiente ya sea dañino o beneficioso. Esa apreciación estará influenciada por la motivación. Las emociones están unidas a las conductas necesarias para la supervivencia como: alimentarse, prevenir enfermedades y accidentes, buscar trabajo, reproducirse, etc. Estas acciones precisan de emociones y motivación.

Rolls (1999), también conecta motivación y emoción y opina que es necesario tener en cuenta procesos como recompensa y castigo para entender las bases neuronales de la emoción y aprender estímulos provenientes del medio ambiente asociado a ellos. Una recompensa esperada que no llega origina un castigo y por el contrario un castigo perdonado crea una recompensa. Creyendo, este autor, que las metas pueden influir en las emociones y en el comportamiento, y que las emociones encierran una motivación para lograr los objetivos.

Las emociones guardan estrecha relación con el conocimiento (memoria, pensamientos, información). Sabemos que la motivación, las emociones y el conocimiento son la base de nuestra conducta.

El estado de vigilia o de alerta que lleva implícito la motivación tiene sus bases neurológicas en el Sistema Nervioso Central, donde se encuentran una serie de estructuras en la corteza cerebral y otras subcorticales que están conexionadas entre sí, es el denominado Sistema Límbico, por otra parte están conectadas con el exterior con los cinco sentidos (vista, oído, olfato, gusto y tacto). Además están presentes unas sustancias neurotransmisoras como la serotonina y la dopamina que producen un aumento del estado de vigilia. El Sistema Límbico es fundamental en la génesis de la motivación.

A partir de la recepción de estímulos, los sentidos envían información a la corteza cerebral y de aquí pasa al Sistema límbico. En el cerebro existen unas sustancias neurotransmisoras como son la serotonina y la dopamina útiles para hacer más rápidas las conexiones. Por otra parte existen unas vías más rápidas que son las tálamolímbicas que no pasan por la corteza cerebral.

Desde el Sistema Límbico los estímulos que van al hipotálamo y tronco encefálico producen la fisiología de las emociones por activación del sistema nervioso simpático y por activación hormonal. Esto creará vasoconstricción o vasodilatación de los vasos arteriales con producción de palidez o rubor respectivamente además de 
respuestas motoras como la huída o la protección y las diferentes expresiones faciales (Armony y col., 1999). El Sistema Límbico interviene en la creación de las diferentes emociones y en la motivación.

La corteza prefrontal (componente del sistema límbico) interviene en la toma de decisiones, en la elección, en la memoria, en la capacidad de atención regulando la profundidad de los sentimientos como se ha demostrado mediante estudios científicos.

Es clásico el ejemplo de lo acontecido en la mitad del siglo XIX a Phineas Gage cuando estando trabajando en el estado de Vermont (U. S. A.) sufrió un accidente y una barra metálica le atravesó el cráneo por la zona prefrontal sobreviviendo al accidente. Gage pasó de ser una persona responsable, amable, educada y trabajadora a convertirse en irresponsable, grosero, voluble en su trabajo y sin capacidad para mantenerse en las tareas.

Damasio (1994a) estudió en profundidad el caso de Gage y también en personas que por accidente, por enfermedad u operación tuviesen esta patología. Aparentemente su razonamiento, inteligencia y memoria seguían en las mismas condiciones, sin embargo aparecían cambios de personalidad, se hicieron antisociales, eran incapaces de planificar el día a día o el futuro. Damasio (1994b), hizo otras pruebas para demostrar que con un estado emotivo se produce una ligera sudoración al realizar juegos que llevaban una apuesta monetaria o al ver fotografías desagradables, mientras no les ocurría a quienes tenían enfermedad en esta zona del cerebro.

Phan y col. (2002), en un metanálisis de numerosos estudios realizados con resonancia magnética funcional en personas normales éstas mostraron como una parte de la corteza prefrontal se activa cuando hay estímulos asociados a ciertas emociones como alegría, tristeza, cólera, miedo o disgusto.

\section{Motivación y Educación Artística}

Los estudios sobre motivación en Educación Artística son escasos, más aún si lo comparamos con las investigaciones sobre motivación en otras áreas educativas como la Educación Física y Expresión Corporal. Esta falta de publicaciones se produce también en los estudios sobre el autoconcepto en el ámbito de la Educación Artística. Los manuales teóricos del área artística, generalmente, aportan recomendaciones o estrategias motivacionales para la enseñanza, sin existir modelos teóricos sobre motivación desarrollados específicamente para la Educación Artística. Posiblemente las investigaciones más avanzadas y específicas sobre motivación y autoconcepto en el ámbito artístico pertenecen al área de Educación Musical (Ortiz e Ibarretxe, 2006; Tripiana, 2010; Valencia, 2011).

\subsection{Las motivaciones en el aula de Educación Artística frente a las motivaciones de la práctica artística externa al marco educativo}

En primer lugar queremos demarcar las motivaciones de la práctica de actividad artística en un contexto educativo y en un ámbito de acción artística libre y personal, dado que muestran diferencias. En la acción artística personal la práctica del arte se 
desarrolla delimitada por las restricciones naturales de los contextos de la persona y sus normas personales. Las motivaciones pueden ser múltiples: canalización del impulso creativo, fines terapéuticos, motivos económicos, mejora de la técnica, etc.

En cambio, en el desarrollo de la Educación Artística en el contexto educativo en el aula, la motivación es una herramienta con la que el profesor trabaja para obtener los mejores resultados posibles de los estudiantes dentro de un contexto curricular, que está definido por objetivos y contenidos académicos adecuados a la edad y características de los alumnos.

En cuanto a la importancia de la motivación en la Educación Artística, González Vida y Maeso Rubio (2005), opinan: “...pensamos que todo futuro profesor o profesora de artes plásticas y visuales debería conocer diferentes estrategias de motivación que puedan utilizarse provechosamente con el alumnado, ya que mediante la estimulación oportuna aumentará el interés de éste por la asignatura, se implicará más activamente en el proceso de aprendizaje, hará una más fácil relectura de la realidad y de sí mismo, obteniendo un mayor conocimiento artístico y unos mejores resultados, siendo las actividades más fructíferas y placenteras para todos/as" (González Vida y Maeso Rubio, 2005:49).

López Salas señala las posibles motivaciones en la creación artística dentro del ámbito educativo: una de ellas sería interna, se trataría del goce estético y satisfacción intelectual que produce la acción creativa en las personas, al margen de los objetivos y el resultado final de la obra, por lo tanto podríamos hablar de una motivación intrínseca que puede proceder del tema, modelo, naturaleza del ejercicio, obras artísticas de referencia, técnicas, conocimientos teóricos, etc. (López Salas, 1999).

A este respecto el mismo autor señala que algunos artistas llegan incluso a perder el sentido del tiempo apoderándose de ellos el proceso creativo. Esta opinión se conecta con la Teoría del flujo o fluir de Csikszentmihályi en que se pierde la noción del tiempo, la tarea es un fin en si mismo y se considera que el esfuerzo realizado es placentero.

Otra motivación que sugiere López Salas (1999), es la obtención de un premio ya sea tangible o no tangible, como halagos del profesor o de la sociedad ante la obra realizada. Por lo tanto consideramos que en la creación artística hay una motivación intrínseca y otra extrínseca.

\subsection{Estudios cuantitativos y cualitativos sobre motivación y Educación Artística}

Como hemos comentado, partimos de una situación caracterizada por el interés de la Psicología hacia la comprensión de los procesos cognitivos en el hecho artístico siendo bajo, hasta el momento, el número de investigaciones específicas sobre aspectos motivacionales en Educación Artística. Hemos agrupado las investigaciones de acuerdo a su carácter en dos grupos:

- Investigaciones que estudian la motivación en Educación Artística dentro de los procesos internos de la actividad artística y en relación con otras variables del proceso. Se trata de las investigaciones más escasas.

- Estudios que aportan estrategias motivacionales para el alumnado y profesorado de Educación Artística. 


\subsubsection{Investigaciones sobre la motivación en Educación Artística en relación con otras variables internas}

Este tipo de estudios de enfoque correlacional aborda las relaciones entre variables como la motivación, la creatividad o el autoconcepto en la Educación Artística.

Dentro de las investigaciones sobre motivación y actividad artística destacamos el estudio de Tripiana Muñoz (2010) dedicado a la relación entre motivación académica y profesional en los alumnos de Estudios Superiores de Música .Tripiana Muñoz halló como factores motivacionales principales la devoción por la música, la ampliación de conocimientos y la aspiración de alcanzar una profesión relacionada con la interpretación musical. El género y la edad influyen en los intereses profesionales, las preferencias por una motivación a corto o largo plazo y en los factores de motivación académica.

Por otro lado también Martín y Martínez (2002), observaron una alta motivación intrínseca en los alumnos de Estudios Superiores de Música y Danza. Rigo y cols. (2010) hallaron en alumnos de Escuela de Arte y Diseño Superior un predominio de motivación intrínseca, así como correlaciones positivas entre inteligencia, creatividad y motivación. Por lo que deducimos que en los estudios profesionales del dominio artístico se combinan, lógicamente, la motivación intrínseca y extrínseca.

Marcos Tello $(1992,1997)$, en su estudio metodológico sobre la motivación en preadolescentes y adolescentes en la Expresión Plástica trabajó con un grupo de niños heterogéneo que acudía una vez por semana a un taller de plástica y en el cual no utilizó refuerzos, económicos ni meritorios, con el fin de evitar la competitividad. Los estímulos externos que se utilizaron fueron: el aula, el grupo social, los materiales y las técnicas, el entorno, las relaciones afectivas y la actitud del profesor. De entre ellos la autora destaca la importancia en los niños de sentirse escuchados y el desarrollar comentarios sobre las obras entre todos los miembros del grupo.

Compartimos con Marcos Tello que la propia Expresión Plástica incluye refuerzos en sí misma: "El proceso como estímulo, el encuentro con el placer entendido placer como objeto-situación deseado. La novedad de la respuesta ante una situación estímulo puede dar nuevas respuestas si se le recompensa por ello: en este caso los refuerzos utilizados pueden encontrarse en la obra final, en la comunicación que aporta en la comprensión de la misma por los adultos y en la propia satisfacción del trabajo" (Marcos Tello, 1992: 223-224).Dentro de las conclusiones finales a las que llega la autora se encuentra que en los preadolescentes se obtienen mejores resultados cuanto mayor es la curiosidad por los materiales y el mobiliario, por lo que una buena motivación en este sentido proporciona que el niño se exprese sin trabas en el medio gráfico-plástico.

Otro estudio realizado en nuestro país es el efectuado por Morales Artero (2001), en alumnos de Educación Plástica y Visual de los cursos comprendidos entre $1^{\circ}$ y $4^{\circ}$ de Educación Secundaria Obligatoria (E.S.O.). Morales Artero (2001), detectó que la motivación disminuye al ascender el curso y era mayor en género femenino que en masculino. Hallándose las valoraciones más altas en las alumnas de $1^{\circ}$ de E. S. O. y las de puntuación más baja en los alumnos de $4^{\circ}$ de E. S. O. Igualmente examinó la competencia percibida de los alumnos, encontrando que al aumentar la edad se manifiesta una autoevaluación más baja. 
Pavlou (2006), y Lekue (2010), han analizado la motivación en niños y preadolescentes. Pavlou (2006) analiza en un pequeño grupo de alumnos de entre 11 y 12 años las relaciones entre las percepciones de competencia o capacidad, motivación y compromiso de los pre-adolescentes en las actividades artísticas. Pavlou también tuvo en cuenta la competencia percibida de los alumnos, concluyendo que la percepción de competencia es un factor clave para determinar el compromiso inicial y el nivel de compromiso con las actividades artísticas.

Los sujetos seleccionados por Pavlou, a la puerta de la adolescencia, muestran interés por el realismo, comienzan a dudar de su capacidad artística y están perdiendo la espontaneidad de etapas anteriores. La investigación quiere indagar en dos cuestiones: la influencia de la concepción de habilidad en el compromiso con las tareas y los tipos de actividades que captan interés en alumnos con bajo nivel de percepción de habilidades. Los resultados hallados en la investigación revelan que cuanto mayor conocimiento en la materia muestra el profesor más actitudes positivas se promueven en los alumnos (Pavlou, 2004 y 2006).

Lekue (2010), analizó las características psicoinstruccionales de preadolescentes y su rendimiento académico en la Educación Artística, concluyendo que existe una relación entre la consecución académica del alumnado y la percepción de disfrute con las tareas artísticas; y también hay relación entre la utilidad de lo realizado en clase, la confianza con las propias habilidades artísticas y el apoyo del profesor. Igualmente comprobó que el compromiso con las tareas artísticas del alumnado disminuye con el aumento de la edad y en cuanto al género las chicas son las que obtienen niveles más altos de apego a la asignatura, como ya había comentado Morales Artero (2001).

\subsubsection{Algunas estrategias motivacionales habituales en el ámbito de la Educación Artística}

Al comienzo de este apartado anticipábamos como los manuales de Educación Artística suelen incluir estrategias y recomendaciones para motivar.

A continuación exponemos una síntesis de algunas de ellas.

\section{A) Estrategias generales para aplicar en los alumnos de Educación Artística}

El esquema de estructuración de una sesión de trabajo en Educación Artística comienza, como en otras materias, con un paso de motivación inicial situado generalmente al comienzo de la clase. Puede tratarse de una reflexión, una lectura, una serie de preguntas lanzadas al aire, o como apunta M. Gata Amaya al referirse al inicio de las clases de educación infantil puede consistir en la observación de un objeto o una imagen que despierte el interés del alumno/a (Gata Amaya, 2003). Por otra parte, la motivación forma parte de todo el proceso de aprendizaje del alumno, siendo el docente mediador en este proceso. El profesor utiliza unas estrategias motivacionales adaptadas por una parte a los contenidos del área, las características de la materia a impartir, o ejercicios a desarrollar y por otra al grupo humano y condiciones en que se desarrollan.

Sabemos que en Educación Plástica al igual que en Educación Musical la capacidad artística se desarrolla por medio de distintas acciones y formas de conducta. En 
la Educación Plástica: observar y desarrollar la sensibilidad perceptiva; representar y crear; interpretar el lenguaje plástico como un sistema estructural (Tolosa, 2003; Gil en Hernández, 1991) y en Educación Musical la audición, la ejecución o la interpretación y la composición (Lacárcel Moreno, 2003).

Eisner (2004), realizó un planteamiento general sobre los conocimientos y aptitudes pedagógicas que necesitaba el profesorado de arte para enseñar adecuadamente y motivar al alumno.

Consideramos que las estrategias motivacionales planteadas por Eisner facilitan además la movilización e interacción de los dos hemisferios del cerebro y el desarrollo de competencias educativas. A continuación exponemos de forma resumida estas estrategias:

- En primer lugar y dada la importancia del uso de la imaginación en el arte, los docentes deberían propiciar el uso de la imaginación en los estudiantes.

- Otros aspectos que requiere la enseñanza del arte son conocimientos sobre materiales y técnicas artísticas, conocimientos sencillos o complejos pero fundamentales en la dinámica del aula.

- Los profesores han de tener la capacidad de leer imágenes, con el fin de leer los trabajos realizados por los estudiantes con una visión pedagógica siempre constructiva que indique al alumno su autoevaluación.

- Los estudiantes deben conocer los objetivos de las actividades, entender el porqué de una actividad o de un proyecto y gozar de un espacio personal en su creación; ahí es donde el profesor ha de entender cómo la interpretación y el punto de vista personal es fundamental en las artes, realizando un seguimiento en las obras de los alumnos sin excederse y permitiendo que la obra sea del alumno.

- El docente debe mostrar en las clases un lenguaje específico y unas actitudes adecuadas a la materia que se está impartiendo y que se va a aprender. Todo ello con un lenguaje claro cuando se explican las técnicas, procesos o significados en la obra.

- Por último es positivo para el alumno que el profesor sepa establecer relaciones entre las obras presentes y las anteriores así como otras referencias artísticas y del mundo exterior.

A estas propuestas para favorecer la motivación en Educación Artística podemos añadir otras como la selección de una temática de interés en los trabajos propuestos concordante con la edad y el nivel educativo de los alumnos (Torrés y Juanola, 1998; González Vida y Maeso Rubio, 2005), y la organización de un adecuado ambiente físico en el aula.

Torrés y Juanola sugieren una serie de elementos a tratar, tanto en observaciones del natural como en temas inventados para las actividades con niños pequeños (Torrés y Juanola,1998:94): la figura humana (yo, familia, amigos, otras personas); los cuerpos del entorno inmediato o imaginario (animales, objetos, plantas); el entorno inmediato o imaginario (ambiente interior o paisaje interior); las escenas vividas 
o imaginarias; la relación entre las personas y la relación entre personas, animales y objetos; lugares donde transcurren las acciones.

Estimamos que los temas planteados son temas básicos susceptibles de ser desarrollados en cualquier nivel educativo con diferentes niveles de complejidad.

Como explican Esquinas y Sánchez (2011), el ambiente físico ideal en que se desarrolle la acción educativa debe estar diseñado para: estimular el sentido estético, fomentar el orden, facilitar la pulcritud y la motivación para desarrollar las tareas. Creándose así un ambiente psicológico en el que se expresen y comuniquen ideas, se desarrollen soluciones a los problemas, se potencie el diálogo y la comprensión.

\section{B) Estrategias motivacionales para los alumnos de Educación Artística en los distintos niveles educativos: niños, adolescentes, adultos y tercera edad}

Las recomendaciones generales que ofrece Eisner se matizarán según el nivel educativo cursado. En Educación Infantil, Fosati (2003), recalca el carácter vivencial y sensorial de la materia, que conjugado con la edad y etapa evolutiva de los niños propicia ampliar el campo perceptivo con una actitud lúdica y creativa. Todo ello en un clima que fomente la libertad creativa y la atención individual del alumno. Oliva y Serrano (2002) destacan el papel de la obra de arte como un elemento generador de interés en los niños de ésta etapa, lo cual consideramos efectivo en todas las edades.

Como ya se ha comentado, un ambiente motivacional positivo en el aula facilitará que los alumnos se involucren. En el trabajo con adolescentes Nuere (2011), considera que las características y conocimientos de partida del alumnado son muy variados, por lo que es importante saber qué es lo que les motiva. Reflexionando sobre la cuestión motivacional en el adolescente la autora plantea dos posibles disposiciones del alumno que el profesor debe captar y resolver: "Puede que no aprendan porque no están motivados" o bien "Puede que, como no aprenden, no se motivan" (Nuere en Esquinas y Sánchez, 2011: 43).

Para motivar a estos estudiantes Nuere sugiere: conocer bien al alumnado, explicar los fines de la materia, aseverar que el aprendizaje es un proceso no necesariamente fácil o agradable, mostrar el error como una parte más del proceso educativo, vislumbrar la motivación como un sentimiento o una postura que nace del interior del alumno, aclarar las aplicaciones y usos que se podrán dar a los contenidos adquiridos y exponer los trabajos realizados para que también puedan ser observados por personas ajenas al aula (Nuere, 2011).

Desde la visión de esta autora en las asignaturas del área no se juega, como en otras, con el temor a suspender porque la sociedad ha concedido poco peso a las asignaturas artísticas. Aunque nosotros opinamos que éste también puede ser un condicionante ya que para algunas familias y alumnos "es imposible" la obtención de calificaciones bajas en Educación Artística, por no decir inadmisible que se suspenda una asignatura considerada aún como "asignatura maría" y de poca utilidad para el estudiante.

Abordar la enseñanza profesional de las artes plásticas en la edad adulta, supone encontrarse con una la falta de planteamientos comunes por parte del profesorado sobre este tema. La docencia en las Facultades de Educación y Formación del 
Profesorado, Bellas Artes y Escuelas Superiores de Arte de España se desarrolla evidentemente según el criterio del docente, y los métodos y estrategias motivacionales utilizadas son propios de cada profesional.

El estudio del valor de la motivación en la Educación Artística con colectivos de la tercera edad elaborado por González Vida y Maeso Rubio (2005), es una investigación interesada por la cuestión realizada en el contexto español. Los autores desarrollaron su experiencia en el aula permanente de formación abierta de la Universidad de Granada. En este caso los alumnos siempre habían estado interesados por realizar prácticas artísticas conociendo técnicas y conceptos propios de la creación artística y aquellas normas necesarias para una representación figurativa. Los docentes tenían en cuenta el valor del factor emocional que sería necesario para desarrollar cualquier actividad artística con personas mayores.

González Vida y Maeso Rubio utilizaron como estrategias motivacionales: el refuerzo positivo, la comunicación horizontal, la evitación de la copia o estereotipo y la introducción de cambios que produjeran efecto de sorpresa.

En su opinión será necesario en el profesor: nociones de psicología de la tercera edad, actitud de ayuda a las personas inseguras y a aquellas que demanden más atención así como conducta emocional de cercanía. También consideran importante que el alumnado atribuya los fracasos y los éxitos al rendimiento y al tiempo dedicado a la tarea, no a su capacidad.

Este último punto se puede resolver analizando conjuntamente entre profesorado y alumnado los trabajos elaborados, valorando lo que se ha realizado correctamente y comentando aquellos aspectos a cambiar o mejorar. Entender que los errores y fracasos se deben a la falta de tiempo dedicado a la tarea o a su grado de dificultad y no a su falta de capacidad o factores externos como la suerte es uno de los aspectos importantes a aclarar en los alumnos (González Vida y Maeso Rubio,2005).

Consideramos que estas pautas también podrían aplicarse en la enseñanza de la Educación Artística con adolescentes y en distintos niveles educativos. Siendo también una cuestión a resaltar la necesidad de utilización de las TIC en el aula como medio de aproximación del alumno a las artes plásticas y a la realidad que le rodea, e igualmente como instrumento de trabajo para la creación. Aspecto éste en el que se están volcando numerosos docentes e investigadores (Arañó, 2002; Escaño, 2010, San Pedro, 2008; Saura, 2011) ante la rápida evolución de las nuevas tecnologías en el contexto educativo.

\section{C) Estrategias para mejorar la motivación en el profesorado de Educación Artística}

Al igual que los estudiantes pueden mejorar su rendimiento por medio de una adecuada motivación, el profesorado puede utilizar estrategias motivacionales para estimular su actividad profesional.

En el trabajo con alumnos de Educación Infantil Torrés y Juanola consideran de gran importancia la carga emotiva del profesor ante los ejercicios de dibujo y pintura, sobre todo cuanto más pequeños son los alumnos, llegando a afirmar: "Si para hacer 
sus trucos los magos necesitan una preparación, un clima y unos artilugios lo mismo sucede a los profesores (...)" (Torrés y Juanola, 1998:96).

El profesorado en la Educación Artística, al igual que en otras áreas necesita trabajo y con ello dedicación, tiempo y energía para convertirse en un mago que esté motivado.

En palabras de Juanola, para el profesor “...no existe mejor motivación que la gratificación que supone el aprendizaje” (Torrés y Juanola, 1998:94), compartimos esta opinión considerando que el desgaste del día a día hace necesaria la aplicación de estrategias motivacionales intrínsecas y extrínsecas en el docente, al igual que en la mayoría de las profesiones.

Secundamos la siguiente opinión de Hernández: “...al igual que los profesores ejercen influencias en cada uno de sus estudiantes y a su vez (y esto suele olvidarse) los estudiantes están influenciando a los profesores" (Hernández, 1991: 73). Por tanto hay una retroalimentación que puede favorecer y mejorar la motivación en ambos.

Finalmente, formulamos algunas recomendaciones generales planteadas por Eisner para motivar a los docentes de arte y con ello superar mejor los obstáculos que se encuentran en la práctica docente, (Eisner, 2004):

1. Una de las más importantes es no caer en la rutina cómoda, ya que esto no conlleva al desarrollo profesional. Por ello se propone afrontar la actividad docente como una forma de investigación personal, como una oportunidad para aprender a enseñar, que rompa con las rutinas y las dinámicas cerradas, favoreciendo una estimulante retroalimentación. Activando una actitud de observación y una postura crítica ante las propias acciones educativas.

2. Otro aspecto es el mantenimiento de una actitud activa o apasionada hacia el área que se está enseñando. Para transmitir pasión, es necesario vivir apasionadamente la asignatura, quizás no siempre llega a ser así, pero evidentemente los estudiantes percibirán el interés y la alegría o por el contrario, el desencanto y la pasividad, con que el docente viva su profesión.

3. Finalmente el cuidado del entorno en que se van a desarrollar las actividades artísticas, así como la ubicación de los estudiantes, los materiales, la interacción de los estudiantes con el medio son otros puntos a tener en cuenta en la docencia de la práctica artística.

\section{Conclusiones}

Todas las estrategias que hemos revisado, tanto las dirigidas al alumnado como al profesorado son propuestas de actitudes y recursos para desarrollar activamente en las aulas, siendo estas sólo una parte de todo el escenario en el que se desarrolla la actividad de la Educación Artística.

Para mejorar la Educación Artística consideramos que es necesario continuar investigando y desarrollando la cuestión motivacional, por medio de investigaciones prácticas en las aulas y de aportaciones de los alumnos y profesores del área, que con su experiencia enriquezcan la teoría y práctica de la motivación en Educación Artística. 


\section{Referencias}

Alonso Tapia, J. (1992). Motivar en la adolescencia. Teoría, evaluación e intervención. Madrid: Universidad Autónoma de Madrid.

Ames, C. y Archer, J. (1988). Achievement goals in the classroom: Students learning strategies and motivation processes. Journal of Educational Psychology, 1992, 80,260-267.

Ames, C. (1992). Classrooms: Goals, structures, and student motivation. Journal of Educational Psychology, 1992, 84, 261-271.

Arañó Gisbert, J.C. (2002). Cibermodernidad o la Educación Artística de Pokemon. Arte, Individuo y Sociedad, 2002, pp.187-194.

Armony J. L., Le Doux, J. E. (1999). How danger is encoded: Towards a systems cellular and computacional understanding of cognitive-emotional interactions in fear circuits. In M.S. Gazzaniga. The Cognitive Neurosciences. MIT Press,Cambridge.

Atkinson,J.W.(1957). Motivationaldeterminantsofrisk-takingbehavior,Psychological Revie, 1957, 64, 359-372.

Berlyne, D.E. (1960). Conflict, arousal and curiosity. New York: McGraw-Hill.

Damasio, A. R. (1994a). Descarte's Error. Londres: Putnam.

Damasio, A. R. (1994b). Descarte's Error and the Future of Human Life. Scientific

American, 271(4): 144.

Damasio, A.R. (1999). The Feeling of What Happens: Body and Emotion in The Making of Conciousness. Londres: Heinemann.

De Volder y Lens, W. (1982). Academic achivement and future time perspective as a Cognitive-Motivational Concept, Journal of Personality and Social Psychology, 1982, 42, 566-571.

Eisner, W. E. (2004). El arte y la creación de la mente. El papel de las artes visuales en la transformación de la conciencia. Barcelona: Paidós Ibérica.

Escaño González, J.C.(2010).Hacia una Educación Artística 4.0. Arte Individuo y Sociedad. Vol.1.,22,pp.13-

Esquinas Pérez, F. y Sánchez Zarco, M. (2011). Didáctica del dibujo: artes plásticas y visuales. Barcelona: Ministerio de Educación.-Graó.

Fosati , A. y Segurado, B. (2003). Expresión plástica y educación infantil. En Caja, La educación visual y plástica hoy, pp.81-123. Barcelona: Graó.

Garrido, I. (1986). La motivación escolar: Determinantes sociológicos del rendimiento. pp. 122-151 en J. Mayor (Dirs.), Sociología y Psicología Social de la Educación. Madrid: Anaya.

Garrido, I. (1990). Motivación, emoción y acción educativa, pp.285-343, en Mayor y Tortosa (Dirs.).

Gata Amaya, M. (2003). La educación plástica en la etapa de infantil: aspectos didácticos y organizativos en el aula. En Domínguez Toscano, (2003) Educación Plástica y Visual hoy. Fundamentos, experiencias y nuevas perspectivas, pp.121134.Océano Ediciones.

Gil en Hernández, M. T. (1991). Consideraciones sobre la Educación Artística. En Hernández, F., Miñarro, A. y Marín Viadel, R. ¿Qué es la Educación Artística? Barcelona: Sendai, pp.45-66. 
González Vida, M. R., y Maeso Rubio, F. (2005). El valor de la motivación en la Educación Artística con personas mayores. Arte, Individuo y Sociedad, 2005. Vol.17, pp.43-60.

González M. C. y Tourón, J. (1992). Autoconcepto y rendimiento escolar. Sus implicaciones en la motivación y en la autorregulación del aprendizaje. Pamplona: EUNSA.

Hernández y Hernández, F., Jódar Miñarro, A., Marín Viadel, R. ( 1991). ¿Qué es la Educación Artística?. Barcelona: Sendai.

Lacárcel Moreno, J. (2003). Psicología de la música y emoción musical. Revista Educatio, 2003 (Diciembre), n 20-21.Diciembre, pp.214-226

Lekue Rodríguez, P. (2010). Características psicoinstruccionales del alumnado preadolescente y rendimiento académico en la Educación Artística escolar. Universidad del País Vasco. Recuperado 10/01/2012 en: http://www.argitalpenak. ehu.es/p291content/eu/contenidos/informacion/se_indice_tescspdf/eu_tescspdf/adjuntos/LEKUE\%20RODRIGUEZ.pdf

Locke, E. (1969). Purpose without consciousness: A contradiction.Psychological Reports, 1969, 21, pp.991-1009.

López Salas, J. L. (1999). Didáctica especifica de la expresión plástica. Oviedo: Universidad de Oviedo.

Marcos Tello, P. (1992), Educación artística y preadolescencia. Revista Interuniversitaria de Formación del Profesorado, 1995, nº 24, (Septiembre a

Diciembre), pp.99-111.

Marcos Tello, P. (1997). Motivación y creatividad en la preadolescencia. Valladolid: Universidad de Valladolid.

Martín, J. y Martínez, R. (2002). Diseño, aplicación y resultados de una encuesta para evaluar aspectos motivacionales en la Escuela Municipal de Música y Danza de San Sebastián. Ponencia II Simposio de la Asociación de Motivación y Emoción. Salamanca, 2002.

Morales Artero, J. J. (2001). La evaluación en el área de educación plástica en la educación secundaria obligatoria. Tesis Doctoral. Barcelona: Universidad Autónoma de Barcelona. Recuperado 26/08/2010 en: http://www.tesisenred.net/ bitstream/handle/10803/5036/jjma01de16.pdf.pdf?sequence $=1$

Nicholls , J. G. (1984). Achievement motivation: Conceptions of ability, subjetive experience, task choice and performance. Pyicholocal Review, 1984, Vol.91, pp.328346

Nuere, S. (2011). La organización del aula: espacio de interacción y comunicación, pp.27-45. En Esquinas Pérez y Sánchez Zarco, Didáctica del dibujo: artes plásticas y visuales (2011). Barcelona: Ministerio de Educación - Graó.

Nuttin, J. (1985). Future time perspective and motivation. Theory and research meth$o d$. Leuven: Leuven University Press y LEA.

Oliva Alcalá, C. y Serrano Llopis, C. (2002).La obra de arte como motivación y aprendizaje en educación infantil, pp.1253-1256. En Barreales Llamas, Moreno Martín y Gervilla Castillo, Necesidades educativas de la infancia ante el nuevo milenio (2002), Vol.2.Málaga: Centro de Ediciones de la Diputación de Málaga (CEDMA). 
Ortiz, H. e Ibarretxe, G. (2006). Formación de profesores para la educación musical y artística: Un estudio comparado. Educación y educadores. Vol. 9, n ${ }^{\circ}$ 2, pp. 33-46.

Pavlou, V. (2004). Profiling primary school teachers in relation to art teaching.Journal of Art and Design Education, 2004, Volume 23,February, Issue, pp.194-204.

Pavlou, V. (2006). Pre-adolescents'perceptions of competence, motivation and engagement in art activities. Journal of Art and Design Education, Volume 25(2),pp.194-204.

Phan, K. L., Wager, T., Taylor, S.F., Liberzon, I. (2002). Funcional Neuroanatomy of Emotion: A Meta-analysis of Emotion Activation Studies in PET and fMRI. Neuroimage, 2002, Vol. 16, pp.331-348.

Reeve, J. (1994). Motivación y Emoción. Madrid: Mc. Graw-Hill

Rigo, D.Y.; Donolo, D.; Ferrándiz García, G. (2010). Laberintos de la mente. Perfil intelectual, creativo y motivacional de alumnos de arte. Revista Anales de Psicología, 2010, vol. 26, no 2 (julio), 2, pp.67-272.Recuperado 20/10/2012 en http://www.um.es/analesps/v26/v26_2/10-26_2.pd

Ross, R. (1975).Saliencie of reward and intrinsic motivation. Journal of Personality and Social Psychology, Vol.32, 245-254.

San Pedro Veledo, J.C. (2008). Pizarras Digitales Interactivas, potencialidades y características. La irrupción de las aulas en la Web 2.0. En Del Moral Pérez y Rodríguez González, Experiencias docentes y tic, pp.229-248, 2008, Oviedo, Octaedro.

Saura, A. (2011). Innovación educativa con TIC en Educación Artística, Plástica y Visual. Líneas de investigación y estudios de casos. Sevilla: MAD, Colección Educación y Psicología.

Super, D. E. (1964). La Psychologie des inteéres. París: Presses Universitarie deFrance.

Tolosa, J. L. (2003). Enseñar la pintura. Guipúzcoa: Universidad del País Vasco

Torrés i Tarrés, M. y Juanola I Argemí, R. (1998). Dibujar: mirar y pensar. Consideraciones sobre educación artística (2 vols).Barcelona: Rosa Sensat.

Tripiana Muñoz, S. (2010). Motivación y orientación profesional en los estudiantes de música. Revista electrónica de motivación y emoción. Vol.13, nº 34.Recuperado 15/02/2011 en http://reme.uji.es/reme/numero34/indexsp.html

Valencia Déniz, R.(2011). Motivación académica en alumnos del Conservatorio Profesional de Música de Las Palmas de Gran Canaria y del Conservatorio de Música de Canarias. Tesis doctoral. Recuperdo 20/12/2012 en http://hdl.handle. net/10553/7111 